\title{
Nucleon Femtography from Exclusive Reactions
}

\author{
Simonetta Liuti ${ }^{* \dagger}$ \\ University of Virginia \\ E-mail: sl4yevirginia.edu
}

Major breakthroughs over the last two decades have led us to access information on how the nucleon's mass, spin and mechanical properties are generated from its quark and gluon degrees of freedom. On one side, a theoretical framework has been developed which enables the extraction of 3D parton distributions from deeply virtual exclusive scattering experiments. On the other hand, the so called gravitomagnetic form factors parameterizing the QCD energy momentum tensor of the nucleon have been connected to the Mellin moments of the 3D parton distributions. Current efforts in both experiment and theory are being directed at using information from electron scattering experiments to map out and eventually visualize these 3D distributions as well as the mass, spin and mechanical properties of the nucleon and of spin 0,1/2, and 1 nuclei. A new science of nucleon femtography is emerging where the 3D structure of the nucleon will be studied merging information from current and forthcoming data from various facilities including the future EIC and the new influx of data science, imaging, and visualization.

23rd International Spin Physics Symposium - SPIN2018 -

10-14 September, 2018

Ferrara, Italy

\footnotetext{
* Speaker.

${ }^{\dagger}$ This work was done under DOE Grant DE-SC0016286
} 


\section{Introduction}

The overarching goal of several experimental programs at Jefferson Lab as well as at the planned Electron Ion Collider (EIC) [1, 2], is to provide an avenue to quantitatively access the spatial 3D quark and gluon structure of the nucleon and to eventually map out its entire phase space/Wigner distribution. Knowledge on both the spatial and momentum distributions of quarks and gluons inside the nucleon will be conducive to understanding, within quantum chromodynamics (QCD), the mechanical properties of all strongly interacting matter including the mass, energy density, angular momentum, pressure and shear force distributions in both momentum and coordinate space. In particular, this will provide a path to ultimately solve the outstanding question of the decomposition of the proton spin into its quark and gluon orbital and spin components.

The key to unlocking direct experimental access to spatial distributions of partons inside the proton was provided by Ji [3], where he suggested Deeply Virtual Compton Scattering (DVCS), $e p \rightarrow e^{\prime} p^{\prime} \gamma$ ( $\gamma$ being a real photon) as a fundamental probe of angular momentum. In DVCS, the high virtuality of the exchanged photon ensures that we are resolving the partonic structure of the proton. At the same time, since it is an exclusive experiment, by measuring the four-momentum transfer between the initial and final proton, $\Delta\left(\Delta^{2}=t\right)$, similarly to elastic scattering experiments, one can obtain information on the location of the partons inside the proton, by Fourier transformation. The hadronic matrix elements for DVCS are described in terms of Generalized Parton Distributions (GPDs), see Refs.[3, 4, 5] and reviews in [6, 7, 8]. GPDs represent a subset of the full 3D parton distributions, the relativistic Wigner phase space distributions [9], that can simultaneously provide information on both the longitudinal momentum fraction, $x$, and the transverse location of partons, $b$, Fourier conjugate to $\Delta$, inside the nucleon. Similarly to the standard (forward) Parton Distribution Functions (PDFs), one can write the Operator Product Expansion (OPE) for the GPDs in terms of twist two operators whose matrix elements are taken between initial and final proton states with different momenta. Using Lorentz symmetry, parity and time reversal invariance, one can write three types of form factors for the spin-n operator associated to electromagnetic (vector) current: $A_{n, 2 i}(t), B_{n, 2 i}(t), C_{n}(t)$, with $i=0,(n-1) / 2$. The latter are identified with the Mellin moments of the GPDs $[3,10]$.

On the other hand, one can parameterize the nucleon matrix element of the Energy Momentum Tensor (EMT), $T^{\mu v}$, stemming directly from the QCD Lagrangian, in terms of 3 conserved form factors: $A_{q, g}, B_{q, g}$ and $C_{q, g}$ [3]. Although the quark and gluon terms are separately renormalization scale dependent, their sum leads to conserved quantities and it is therefore apt to represent the mechanical properties of hadronic matter. Similarly to the definition of the classical EMT, the QCD EMT time components encode the densities and flux densities of the quark and gluon fields energy and momentum, so that by integrating over the volume and summing over the quark and gluon components, one obtains the system's total energy and momentum from the time components, $T^{0 i}$, while the space, $T^{i j}$, elements can be identified with the pressure $(i=j)$, and the shear forces $(i \neq j)$. Evaluating the EMT matrix elements one finds that $A_{q, g}(t=0)$ represents the total quark/gluon longitudinal momentum relative to the nucleon momentum. By Fourier transformation, the form factor, $A_{q, g}(t)$, therefore provides information on how the momentum is spatially distributed inside the nucleon. Similarly, $t C_{q, g}(t)$ is identified with the pressure density distribution in the nucleon $[11,12,13,14,15]$. The combination $A_{q, g}(t)+B_{q, g}(t)$ gives the quark and gluon contributions to 
the proton angular momentum [3].

In Ref.[3], Ji took the fundamental step of linking the information from the GPDs OPE form factors for $n=2$, accessible in DVCS type experiments, and the elements of the QCD EMT, by showing that:

$$
A_{20}^{q, g}=A_{q, g}(t), \quad B_{20}^{q, g}=B_{q, g}(t), \quad C_{20}^{q, g}=4 C_{q, g}(t) .
$$

This important step enables investigations of the mechanical properties of the nucleon by extracting GPDs from dedicated exclusive high energy electron scattering experiments.

While we can benefit from a methodology fully developed through decades to extract Parton Distribution Functions (PDFs) from a variety of inclusive scattering experiments, the establishment of a similar quantitative program for GPDs has just begun. Stating the problem in a nutshell, to obtain the important additional information on the 3D spatial structure of parton distributions encoded in GPDs requires a variety of deeply virtual coincidence experiments, which, at variance with their inclusive counterparts, bring in additional complications. To mention a few phenomenology issues: GPDs appear in the cross section embedded in complex amplitudes (the so called Compton Form Factors, CFFs); the cross section has a complicated dependence on the phase measured by the azimuthal angle, $\phi$, between the lepton and hadron planes; due to the enhanced kinematic complexity, it is harder to separate out the leading and subdominant components in the scale, $Q^{2}$. Pioneering experiments conducted mostly at HERMES and Jefferson Lab have shown that GPDs extraction from data is potentially at reach [8]. An impressive effort is underway to address the aforementioned complications. In particular, this will require going beyond the standard computational toolbox and addressing the computational challenges that are presently limiting our access to the proton's 3D structure. The Center for Nucleon Femtography (CNF) [16] was recently founded at Jefferson Lab to lead and coordinate efforts in this direction.

\section{The QCD Energy Momentum Tensor and Generalized Parton Distributions}

The QCD EMT is defined as,

$$
T_{Q C D}^{\mu v}=\bar{\psi} \gamma^{(\mu} \overleftrightarrow{D}^{v)} \psi+F^{\mu \alpha} F_{\alpha}^{v}+\frac{1}{4} g^{\mu v} F^{2}
$$

where $\psi$ and $F^{\mu \alpha}$ are the quark and gluon fields, respectively, while $g^{\mu v}$ is the spacetime metric. The EMT matrix element between nucleon states was parameterized in Ref.[10] as,

$$
\begin{aligned}
\left\langle p^{\prime}\left|T_{q, g}^{\mu v}\right| p\right\rangle & =\bar{U}_{s^{\prime}}\left(p^{\prime}\right)\left[A_{q, g}(t) \gamma^{(\mu} P^{v)}+B_{q, g}(t) \frac{P^{(\mu} i \sigma^{v) \rho} \Delta_{\rho}}{2 M}\right. \\
& \left.+\frac{1}{4 M} C_{q, g}(t)\left(\Delta^{\mu} \Delta^{v}-g^{\mu v} \Delta^{2}\right)+\bar{C}_{q, g}(t) g^{\mu v} M\right] U_{s}(p),
\end{aligned}
$$

where $q$ and $g$ are the quark and gluon labels; $M$ is the nucleon mass; the initial (final) nucleon spinor is $U_{s}(p)\left(\bar{U}_{s^{\prime}}\left(p^{\prime}\right)\right) ; P=\left(p+p^{\prime}\right) / 2$, and the momentum transfer is, $\Delta=p^{\prime}-p, t=\Delta^{2}<0$. Taking the light cone components in Eq.(2.2) leads to the momentum and angular momentum sum rules, respectively given by,

$$
\begin{gathered}
\sum_{i=q, g} A_{i}=\varepsilon_{q}+\varepsilon_{g}=1 \\
\frac{1}{2} \sum_{i=q, g}\left(A_{i}+B_{i}\right)=J_{q}+J_{g}=\frac{1}{2} .
\end{gathered}
$$


where $\varepsilon_{q, g}$ denote the fractions of the proton momentum carried by quarks and gluons. These basic constructs of the theory in turn can be accessed experimentally owing to their connection, through the operator product expansion (OPE), to the Mellin moments of specific parton distributions which parameterize both the forward $\left(p=p^{\prime}\right)$ and off-forward $\left(p \neq p^{\prime}\right)$ quark and gluon correlation functions. The completely unintegrated off forward quark-quark correlation function is defined as the matrix element between proton states with momenta and helicities $p, \Lambda$ and $p^{\prime}, \Lambda^{\prime}$,

$$
W_{\Lambda^{\prime} \Lambda}^{\Gamma}(P, k, \Delta ; \mathscr{U})=\frac{1}{2} \int \frac{d^{4} z}{(2 \pi)^{4}} e^{i k \cdot z}\left\langle p^{\prime}, \Lambda^{\prime}\left|\bar{\psi}\left(-\frac{z}{2}\right) \Gamma \mathscr{U} \psi\left(\frac{z}{2}\right)\right| p, \Lambda\right\rangle,
$$

where $\mathscr{U}$ is the gauge link connecting the quark operators at positions $-z / 2$ and $z / 2, \Gamma$ is a Dirac structure, $\Gamma=\mathbf{1}, \gamma^{5}, \gamma^{\mu}, \gamma^{\mu} \gamma^{5}, i \sigma_{\mu \nu}$, and $k$ is the quark four-momentum. The Generalized Parton Distributions (GPDs) are obtained by formally integrating Eq.(2.5) over $k^{-}$and the transverse parton momentum, $k_{T}$, provided that the gauge link has the appropriate form (see discussion in Ref.[41]). For the leading order electromagnetic interaction/vector case the operator is $\Gamma=\gamma^{+}$, and one can parameterize the correlation function as,

$$
W_{\Lambda^{\prime} \Lambda}^{\gamma^{+}}=\frac{1}{2 P^{+}} \bar{U}\left(p^{\prime}, \Lambda^{\prime}\right)\left[\gamma^{+} H+\frac{i \sigma^{+\Delta}}{2 M} E\right] U(p, \Lambda)=H(x, \xi, t)\left(\delta_{\Lambda, \Lambda^{\prime}}+\frac{\left(\Lambda \Delta^{1}+i \Delta^{2}\right)}{2 M} E(x, \xi, t) \delta_{-\Lambda, \Lambda^{\prime}}\right.
$$

$H(x, \xi, t)$ and $E(x, \xi, t)$ are the GPDs which depend on the longitudinal momentum transfer between the initial and final proton, represented through the skewness parameter $\xi$, and the fourmomentum transfer squared, $t ; x$ is the light cone momentum fraction carried by the parton [7, 6]. In particular, $H_{q}(x, 0,0) \equiv q(x), H_{g}(x, 0,0) \equiv g(x)$, where $q(x)$ and $g(x)$ are the unpolarized quark (antiquark) and gluon distributions. From the structure of the correlation function one can immediately see that the second Mellin moment obtained by taking the derivative with respect to the longitudinal variable in Eq.(2.5),

$$
\left\langle p^{\prime}\left|\bar{\psi}(0) \gamma^{+} i D^{+} \psi(0)\right|\right\rangle=2 P^{+} \int_{1}^{1} d x x H_{q}(x, \xi, t)
$$

is equivalent to the matrix element of the quark contribution to the EMT in Eq.(2.2), Eq.(2.11), leading to the momentum sum rule,

$$
A_{q, g}(t)=\int_{0}^{1} d x x H_{q, g}(x, 0, t) \equiv A_{20}^{q, g}(t)
$$

A similar derivation allows us to write angular momentum in terms of moments of GPDs as,

$$
A_{q, g}(t)+B_{q, g}(t)=\int_{0}^{1} d x x\left(H_{q, g}(x, 0, t)+E_{q, g}(x, 0, t)\right) \equiv A_{20}^{q, g}(t)+B_{20}^{q, g}(t) .
$$

For $t=0$, Eq.(2.9) leads to the Ji sum rule [3].

$A_{q}(0)$ has been known from deep inelastic scattering experiments for a long time since it represents the quark average longitudinal momentum fraction. Its $t$ dependence, as well as the form factor $B_{q}(t)$ have been extracted in first pioneering model dependent studies (see e.g. [17] and references therein). The gluon moments have not yet been obtained in experiment. 
While the focus of experimental and theoretical investigations has been mostly on the angular momentum sum rule, more recent developments address the EMT form factors $C_{q, g}$ [23] and $\bar{C}_{q, g}$, [27], respectively. Similarly to how $A_{q, g}$ and $B_{q, g}$ are associated with the energy, momentum and angular momentum distributions inside the proton, the $C_{q, g}$ form factor (also know as the $D$-term $[6,7])$ describes the internal mechanical properties of pressure and shear distributions and it is obtained from the spatial components of the EMT [18, 11, 12, 13, 14],

$$
T_{i j}(\vec{r})=\frac{1}{M} \int \frac{d^{3} \Delta}{(2 \pi)^{3}} e^{i \vec{q} \cdot \vec{r}}\left(\Delta_{i} \Delta_{j}-\Delta^{2} \delta_{i j}\right) C_{q, g}(t)
$$

$C_{q, g}$ is defined as the coefficient of the skewness dependent part of the second Mellin moment which reads,

$$
\int_{0}^{1} d x x H_{q, g}(x, \xi, t) \equiv A_{20}^{q, g}(t)+\left(2 \xi^{2}\right) C_{2}^{q, g}
$$

$C_{q}$ has also been recently been extracted from DVCS experiments at Jefferson Lab, [19]. $C_{g}(t)$ has not been measured. While various lattice QCD evaluations of $A_{q}, B_{q}$, and $C_{q}$ have been performed by several groups [20, 21, 22] their gluon counterparts have just recently been evaluated in Ref.[23]. Knowing the pressure and energy-momentum spatial distributions inside the proton from the matrix elements of the QCD EMT between nucleon states has profound consequences. In particular, one can obtain constraints on the Equation of State (EoS) for the quark matter phase in hybrid neutron stars: through our QCD description of the EMT, the observables from the binary neutron stars merger GW170817 [24] could be confronted with laboratory measurements from electron scattering experiments [25]. In particular, the QCD-EMT-based underlying description allows us to evaluate the so far elusive gluon contribution to the EoS. In Fig.1 the quark-gluon contribution to the EoS constructed from the QCD EMT is shown along with different predictions and with the region allowed by the LIGO constraint from the GW170817 binary neutron star merger.

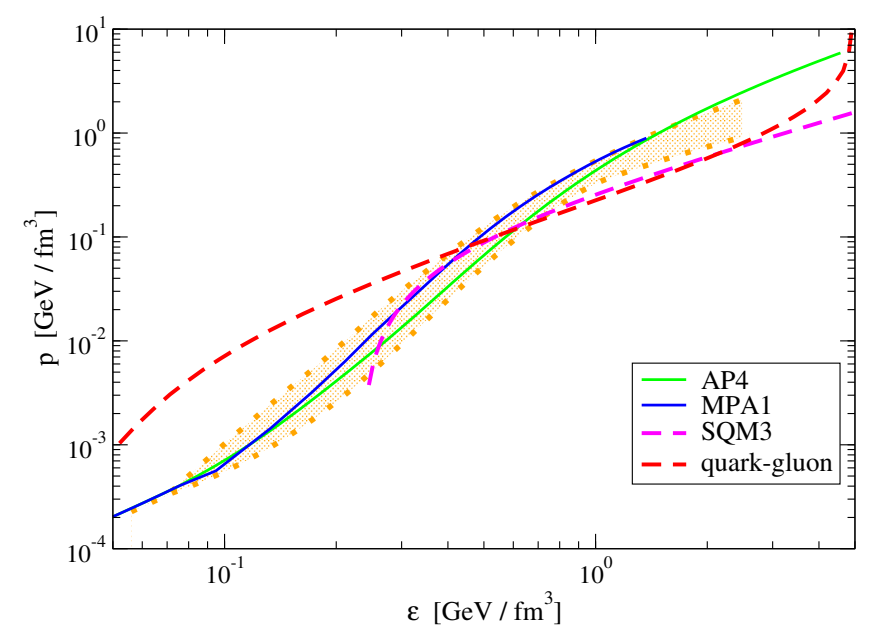

Figure 1: Adapted from Ref.[25] The EoS of neutron stars. The shaded region is the allowed region from GW170817; (red dashed) the quark-gluon EoS constructed from the QCD EMT; two hadronic EoS AP4 (green solid) and MPA1 (blue solid); (magenta dashed) quark matter EoS SQM3. 
The term, $\bar{C}_{q, g}$, is the most elusive one since it is not associated with any GPD. In Refs.[26, 27] however, $\bar{C}_{g}$ was evaluated and shown to contribute to the twist-four operator $F^{2}$, Eq.(2.2), or to the trace anomaly. This term can be measured in exclusive production of heavy quarkonium states such as $J / p s i$ at threshold in electron-proton scattering ([28] and references therein).

Spin 0 nuclei are extremely interesting to study in this context because they allow us to focus on the pressure and energy terms without complications due to the nucleon spin. The EMT for a spin 0 system (for instance the ${ }^{4} \mathrm{He}$ nucleus) reads,

$$
\left\langle p^{\prime}\left|T_{q, g}^{\mu v}\right| p\right\rangle=\left[A_{q, g}(t) P^{\mu} P^{v}+\frac{1}{4 M} C_{q, g}(t)\left(\Delta^{\mu} \Delta^{v}-g^{\mu v} \Delta^{2}\right)+\bar{C}_{q, g}(t) g^{\mu v} M\right],
$$

$C_{q, g}(t)$ was evaluated in ${ }^{4} \mathrm{He}$ in Ref.[29] using a realistic microscopic model of nuclear GPDs. Nuclear modifications were shown to affect this term proportionally to the nuclear separation energy $E$, namely, in a spin 0 nucleus with $A$ nucleons, $C_{q, g}(t) \propto A(1-E / M)$ (this is at variance with the steeper growth predicted by the liquid drop model based, back of the envelope calculation of [13]). Experimental measurements to disentangle this term are described in the ALERT proposal [30].

Finally, spin 1 systems such as the deuteron give a richer range of form factors for the EMT, essentially due to terms associated to the quadrupole moment, and to new GPDs associated to the deuteron tensor structure. The complete parameterization of the EMT tensor was described in Ref.[31], while the parameterization of the deuteron vector and axial vector correlation function in terms of GPDs was initially given in Ref.[32]. It follows that, similarly to the nucleon case, by taking the second moments with respect to $x$ of the deuteron GPDs one can find relations between the second moments of the GPDs $H_{i}$ and the deuteron EMT form factors $\mathscr{G}_{i}(i=1,7)[31]$,

$$
\begin{aligned}
& 2 \int d x x\left[H_{1}(x, \xi, t)-\frac{1}{3} H_{5}(x, \xi, t)\right]=\mathscr{G}_{1}(t)+\xi^{2} \mathscr{G}_{3}(t) \\
& 2 \int d x x H_{2}(x, \xi, t)=\mathscr{G}_{5}(t) \\
& 2 \int d x x H_{3}(x, \xi, t)=\mathscr{G}_{2}(t)+\xi^{2} \mathscr{G}_{4}(t) \\
& -4 \int d x x H_{4}(x, \xi, t)=\xi \mathscr{G}_{6}(t) \\
& \int d x x H_{5}(x, \xi, t)=-\frac{t}{8 M_{D}^{2}} \mathscr{G}_{6}(t)+\frac{1}{2} \mathscr{G}_{7}(t)
\end{aligned}
$$

Based on counting of the $J^{P C}$ quantum numbers in the t-channel (Figure 2 and Ref.[33]), the EMT admits 7 conserved deuteron form factors (see discussion in [34]). The physical meaning of $\mathscr{G}_{5}(t)$ entering Eq.(2.14) is particularly interesting since it connects partonic angular momentum in the deuteron, $J_{z}$, with the GPD $H_{2}$, which represents the magnetic response,

$$
\left\langle p^{\prime}\left|\int d^{3} x\left(\vec{x} \times \vec{T}_{q, g}^{0 i}\right)_{z}\right| p\right\rangle=G_{5,2}(0) \int d^{3} x p^{0} \Rightarrow G_{5,2}(0)=2 J_{z}^{q}
$$

\subsection{OAM and other generalized Wandzura Wilczek relations}

An extensive amount of literature has been dedicated to understanding how the quark and gluon angular momentum is partitioned into its spin and orbital components. While the momentum 


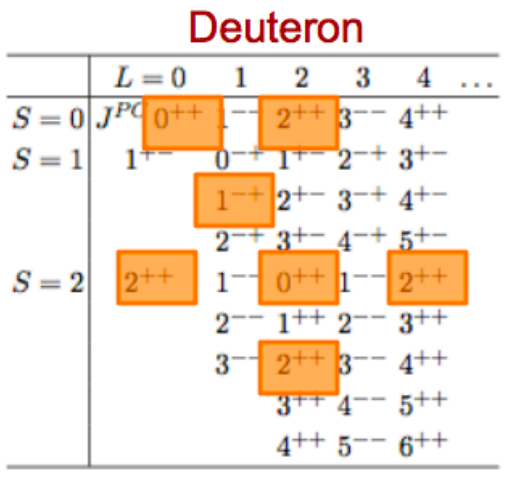

TABLE II: $J^{P C}$ of the $d \bar{d}$ states.

Figure 2: Adapated from [33]. $J^{P C}$ quantum numbers of the $d \bar{d}$ states.

sum rule has an immediate dynamical interpretation in terms of the average longitudinal momentum carried by the different parton components, to obtain a dynamically transparent expression for the angular momentum sum rule one has to break it down into its spin and Orbital Angular Momentum (OAM) components, while simultaneously preserving the gauge invariance of the theory. The decomposition can be performed within two different approaches, by Jaffe and Manohar (JM) [35],

$$
\frac{1}{2} \Delta \Sigma_{q}+L_{q}^{J M}+\Delta G+L_{g}^{J M}=\frac{1}{2}
$$

and by $\mathrm{Ji}[3]$,

$$
\frac{1}{2} \Delta \Sigma_{q}+L_{q}^{J i}+J_{g}^{J i}=\frac{1}{2}
$$

The JM longitudinal OAM distribution has been identified with a parton Wigner distribution weighted by the cross product of position and momentum in the transverse plane, $b_{T} \times k_{T}$ [36]. Parton Wigner distributions can be related, through Fourier transformation, to specific Generalized Transverse Momentum-Dependent Parton Distributions (GTMDs), which are off-forward TMDs. The correlation defining OAM corresponds to the GTMD $F_{14}$ (we follow the naming scheme of Ref.[37]). In particular, the OAM distribution is described by the $x$-dependent $k_{T}^{2}$ moment of $F_{14}$. The difference between JM and Ji's decompositions arises from the way in which the gauge invariance of the theory intervenes through the gauge link in the relevant parton correlator from which $F_{14}$ is extracted [38]. Ji OAM results from a straight gauge link, whereas JM OAM results from a staple-shaped gauge link such as the one used in the standard definition of TMDs. In Ref.[39], in particular, it was shown that JM OAM, $L_{q}^{J M}$, can be written as the sum of Ji's OAM, $L_{q}^{J i}$, plus a matrix element including the gluon field. While $J_{q, g}$ measurements through GPDs are in progress, GTMDs have, however, only been evaluated in ab initio calculations [40], since in order to disentangle the $k_{T}$ and $b_{T}$ (or $\Delta_{T}$ ) directions require so far prohibitive exclusive measurements of two outgoing particles in the two distinct hadronic planes.

Refs.[41, 42] were dedicated to defining observables that would enable direct access to OAM in experimental measurements. In particular, the following relations which combine information from 
Lorentz Invariant Relations (LIRs) and QCD Equations of Motion (EoM) relations, allow us to write the GTMD defining OAM in terms of a twist-3 GPD, $\tilde{E}_{2 T}$,

$$
L_{z}^{q}(x)=-\int d^{2} k_{T} \frac{k_{T}^{2}}{M^{2}} F_{14}=\int_{x}^{1} d y\left(\widetilde{E}_{2 T}+H+E+\mathscr{A}_{F_{14}}\right)
$$

where we took the forward limit $(\xi \rightarrow 0) ; \mathscr{A}_{F_{14}}(x)$ is a term containing the gauge link dependent, or quark-gluon-quark, components of the correlation function (for a straight gauge link, $\mathscr{A}_{F_{14}}(x)=0$ ). Other relations important for OAM are,

$$
\begin{gathered}
\left(L_{z} S_{z}\right)_{q}(x)=\int d^{2} k_{T} \frac{k_{T}^{2}}{M^{2}} G_{11}=\int_{x}^{1} d y\left(2 \widetilde{H}_{2 T}^{\prime}+E_{2 T}^{\prime}+\widetilde{H}-\mathscr{A}_{G_{11}}\right) \\
\int d^{2} k_{T} \frac{k_{T}^{2}}{M^{2}} F_{12}^{o} \equiv-f_{1 T}^{\perp(1)}=-\left.\mathscr{M}_{F_{12}}\right|_{\Delta_{T}=0}
\end{gathered}
$$

Eq.(2.22) represents the spin-orbit contribution, while in Eq.(2.23), the $l h s$ is an off-forward generalization of the Sivers shift [43, 44, 45], while the term $\mathscr{M}_{F_{12}}$ on the rhs is an off-forward/generalized analogue of the Qiu-Sterman $T_{q}(x, x)$ term [46].

\section{Femtography}

The exploration of the 3D structure of the nucleon including its mechanical properties encoded in the EMT has recently given origin to the science of Nuclear Femtography. Nuclear Femtography is probed in various deeply virtual exclusive reactions: an important role is played by studying the response of the system to polarization of both the electron beam and of the nucleon and nuclear targets. The study of femtography implies a profound reorganization of our approach to exploring the internal structure of the nucleon. This step up in phenomenological methodologies is required by the complexity of the processes which is translated into many more variables that need to be simultaneously accounted for in high energy coincidence reactions. An additional set of complications arises in extracting the various observables which appear in the cross section at the amplitude level. A new generation of current and planned experiments at the future EIC [1] could in principle allow us to incorporate all the information from data and phenomenology into a tomographic image connecting the deepest part of the quantum world with what we see as everyday matter around us. However, to harness and organize information from experiment and increase the reach of this emergent field will require going beyond the standard computational toolbox.

The Center for Nuclear Femtography [16] was recently created at Jefferson Lab to address this diverse set of issues. On one side, there is a need for a new computational and visualization effort to examine and evaluate the use of new state of the art computational methods and techniques, including visualization to address the many layers of analysis which are necessary to extract the signal in its complex background after the large experimental data sets are acquired. On the other, several theoretical issues are being addressed from modeling the 3D structure to providing a full description of the cross sections for the various deeply virtual exclusive processes is needed.

An essential starting point is to provide the formalism and theoretical framework for deeply virtual exclusive-type experiments including DVCS, Deeply Virtual Meson Production, $e p \rightarrow e^{\prime} p^{\prime} M$ (DVMP), and Timelike Compton Scattering (TCS), $\gamma p \rightarrow l^{+} l^{-} p^{\prime}$, where a large invariant mass lepton pair is produced (recently reviewed in [8]). Only by measuring the polarization of the beam 
(including the photon beam in TCS), of the target and outgoing protons will provide an adequate number of observables to extract the various Compton Form Factors containing information on GPDs. For a reliable extraction and interpretation of physics observables from experiment it is important to introduce the formalism for all deeply virtual exclusive processes according to an agreed-upon-by-the-community set of benchmarks

A complete formalism for the photon electroproduction cross section including DVCS and the interference with the Bethe-Heitler (BH) process was recently provided in Ref.[47]. The new formalism is a step up from the formalism adopted in DVCS analyses so far which was describing the cross section through an approximate series in harmonics of the azimuthal angle, $\phi$. The formalism to treat coincidence reactions is based on a generalized Rosenbluth-type description [48]. This formulation, while emphasizing the physics content of the various contributions, e.g. by making a clear parallel with coincidence scattering experiments, introduces more complex $\phi$ dependent kinematic coefficients than the harmonics based prescription. This level of precision is a must in order to extract GPDs from DVCS analyses in order to disentangle the twist two contributions from transversity gluons and higher twist terms. The price of evaluating more complex $\phi$ structures is payed off not only by having a much clearer physics-based formulation, but also by the fact that the coefficients are exactly calculable. For illustration, we show the contributions of the BH and BH-DVCS interference term to the unpolarized cross section,

$$
\begin{aligned}
& \frac{d^{5} \sigma_{\text {unpol }}^{B H}}{d x_{B j} d Q^{2} d|t| d \phi d \phi_{S}}=\frac{\Gamma}{t^{2}}\left[A_{B H}\left(F_{1}^{2}+\tau F_{2}^{2}\right)+B_{B H} \tau G_{M}^{2}(t)\right] \\
& \frac{d^{5} \sigma_{\text {unpol }}^{\mathscr{I}}}{d x_{B j} d Q^{2} d|t| d \phi d \phi_{S}}=\frac{\Gamma}{Q^{2}(-t)}\left[A_{\mathscr{I}}\left(F_{1} \Re e \mathscr{H}+\tau F_{2} \Re e \mathscr{E}\right)+B_{\mathscr{I}} G_{M} \Re e(\mathscr{H}+\mathscr{E})+C_{\mathscr{I}} G_{M} \Re e \widetilde{\mathscr{H}}\right] .
\end{aligned}
$$

The detailed equations are derived and discussed in Ref.[47] (for illustration purposes, only the unpolarized case for BH (Eq.(3.1)) and the unpolarized leading order for the BH-DVCS interference term (labeled $\mathscr{I}$ in Eq.(3.2)), are quoted).

In both equations, $F_{1}, F_{2}$ are the Dirac and Pauli form factors, $G_{M}=F_{1}+F_{2}$ is the magnetic form factor $\left(G_{E}^{2}=F_{1}^{2}+\tau F_{2}^{2}\right) ; t$ is the momentum transfer squared, $\left(\tau=-t / 4 M^{2}\right)$; in Eq.(3.2) $\mathscr{H}, \mathscr{E}, \widetilde{\mathscr{H}}$ are Compton form factors containing the GPDs that integrate to $F_{1}, F_{2}$ and $G_{A}$, respectively [6]. $A_{B H}, B_{B H}$ are kinematic coefficients which are exactly calculable and rendered in covariant form; $A_{\mathscr{I}}, B_{\mathscr{I}}, C_{\mathscr{I}}$ are also covariant kinematic coefficients which, however, contain an extra dependence on the phase $\phi$ as we also explain in what follows. The new formalism allows one to emphasize the physics content of the cross section: in both Eq. (3.1) and Eq.(3.2) we can identify the first term with the electric form factor type contribution, and the second term with the magnetic form factor contribution. For the BH-DVCS interference we also have an extra function which includes the axial GPD (interestingly, a similar term would also be present in BH but parity violating).

In Figure 3 we illustrate the working of the Rosenbluth separation, Eq.(3.1), for one of the kinematic settings from the Jefferson Lab experiment E00-110 [49]: on the left panel we show the $e p \rightarrow e^{\prime} p^{\prime} \gamma$ unpolarized cross section data plotted vs. $\phi$; on the right panel we plot the reduced cross section for the same set of data vs. the kinematic variable, $A_{B H} / B_{B H}$ (the detailed definition 

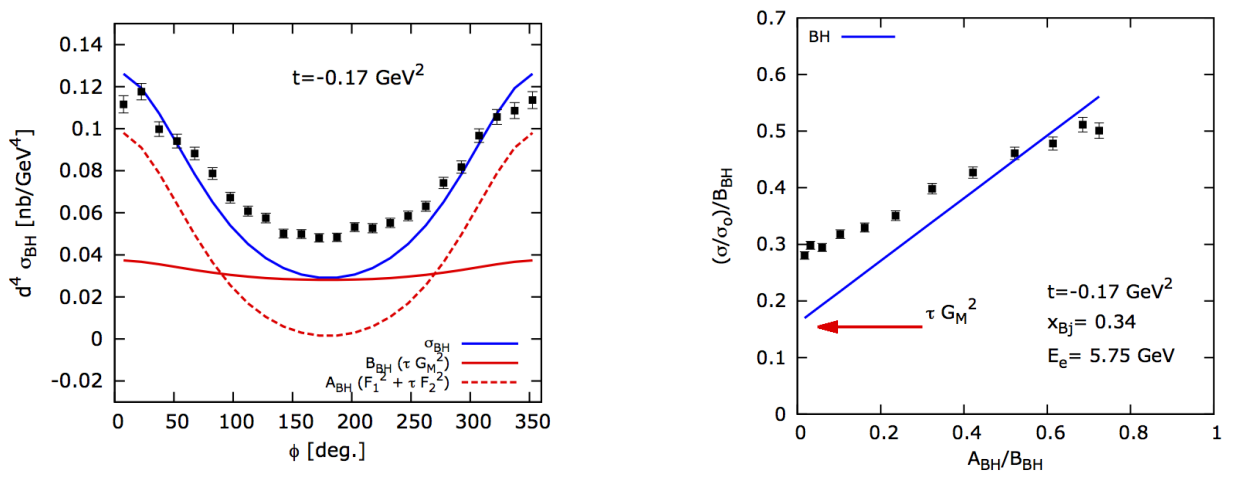

Figure 3: Adapted from Ref.[47]. Left: A sample of the new precise $e p \rightarrow e^{\prime} p^{\prime} \gamma$ data from Ref.[49] for unpolarized lepton proton scattering in the kinematic setting: $E_{e}=5.75 \mathrm{GeV}, Q^{2}=1.82 \mathrm{GeV}^{2}, x_{B j}=0.34$, $t=-0.172 \mathrm{GeV}^{2}$. The cross section is plotted vs. the azymuthal angle, $\phi$. The curves represent the $\mathrm{BH}$ contribution: (red dashed line) $\tau G_{M}^{2}$ term; (red full line) $\left(F_{1}^{2}+\tau F_{2}^{2}\right)$ term; (blue) sum of the two; Right: Reduced cross section obtained from the same set of data plotted vs. the kinematic variable $A_{B H} / B_{B H}$ from the Rosenbluth-type formula, Eq.(3.1). The straight line represents the $\mathrm{BH}$ calculation intercepting the y-axis at $\tau G_{M}^{2}$.

of this quantity is given in [47]). The $\mathrm{BH}$ cross section appears as a linear function of the variable $A_{B H} / B_{B H}$, with intercept given by $\tau G_{M}^{2}$ and slope given by $F_{1}^{2}+\tau F_{2}^{2}$. The difference between the data and the $\mathrm{BH}$ line reflects the contribution from the DVCS process.

A generalized Rosenbluth separation can be performed for the BH-DVCS interference case, Eq.(3.2), by defining an analogous kinematic variable, $A_{\mathscr{I}} / B_{\mathscr{I}}$ similar to $A_{B H} / B_{B H}$ defined for BH. As it can be surmised from Eq.(3.2), if one groups the small $C_{\mathscr{I}}$ coefficient with the $A_{\mathscr{I}}$ term, the intercept with the y-axis is given by $\tau G_{M} \Re e(\mathscr{H}+\mathscr{E})$. We illustrate this situation in Figure 4.

Similar structures are found for other polarization configurations. In particular, the careful analysis conducted in [47] allows us to disentangle all the twist three contributions: among these in particular, is the GPD $\widetilde{E}_{2 T}$ which measures OAM (see Section 2.1). Therefore: OAM can be extracted directly from deeply virtual exclusive electroproduction data.

\section{Conclusions and Outlook}

In summary, the study of GPDs has opened a new frontier in QCD providing a new paradigm that will allow us to both penetrate and visualize the deep structure of visible matter and to answer so far inaccessible questions on the spatial distribution of subatomic matter. Simultaneously, by connecting the GPD Mellin moments to the QCD gravitomagnetic form factors provides experimental access to exploring the nature of mass, spin and the distribution of the strong force. This 

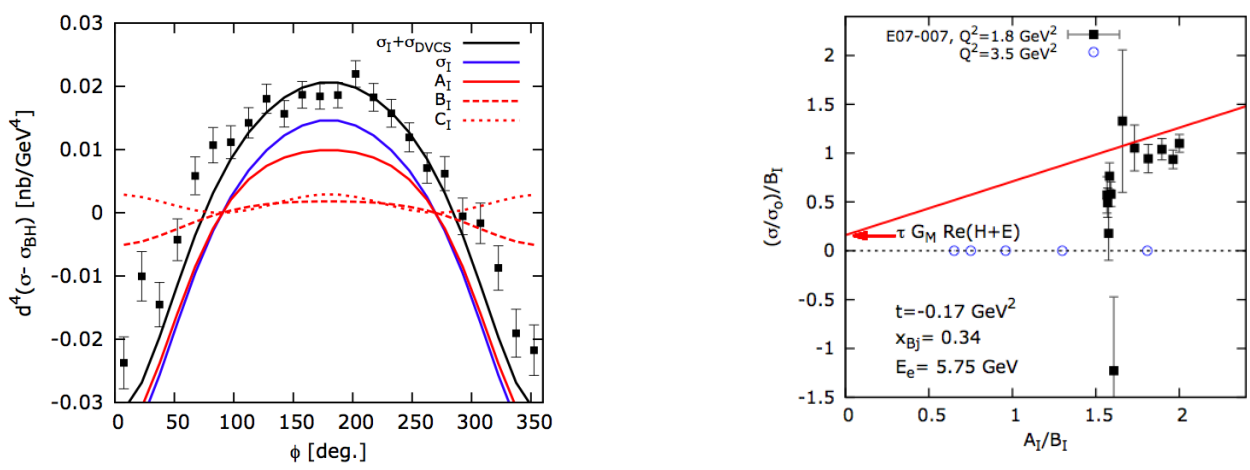

Figure 4: Preliminary results on the generalized Rosenbluth separation performed for the BH-DVCS interference term displayed in Eq.3.2. Left: difference of the $e p \rightarrow e^{\prime} p^{\prime} \gamma$ unpolarized cross section data from Ref.[49] in the kinematic setting: $E_{e}=5.75 \mathrm{GeV}, Q^{2}=1.82 \mathrm{GeV}^{2}, x_{B j}=0.34, t=-0.172 \mathrm{GeV}^{2}$, and the calculated $\mathrm{BH}$ cross section; the blue line is a model calculation of the interference term; the red lines represent the separate contributions of the terms with coefficients $A_{\mathscr{I}}, A_{\mathscr{I}}$ and $A_{\mathscr{I}}$, respectively; the black line is the sum of the BH-DVCS interference and the DVCS modulus squared model calculations. Right: Reduced cross section for the BH-DVCS interference term obtained from the same set of data in the Left panel plotted vs. the kinematic variable $A_{I} / B_{I}$ from the Rosenbluth-type formula, Eq.(3.2). The straight line represents the BH-DVCS interference calculation intercepting the y-axis at $\tau G_{M}(\mathscr{H}+\mathscr{E})$. For this kinematics the data points are clustered away from the y-axis, making the determination of this term more uncertain. The open blue points are a kinematic evaluation of the $A_{I} / B_{I}$ values obtained by choosing a higher value of $Q^{2}$, $Q^{2}=3.5^{2}$ for the same values of $E_{e}, x_{B j}$ andt. This example shows that by varying $Q^{2}$ one obtains a better coverage towards low values of $A_{\mathscr{I}} / B_{\mathscr{I}}$, which wold allow a precise extraction of the angular momentum term from data.

step revolutionizes our way of thinking phenomenology, putting it in a central place to address physics questions previously accessed only through "thought of" experiments in lattice QCD. Furthermore, GPDs represent a window into Wigner distributions. To observe, evaluate and interpret Wigner distributions will require stepping up data analyses from the standard methods adopted so far, to developing new numerical/analytic/quantum computing methodologies. Unprecedented discoveries will be at reach by harnessing information from these new analyses within a concerted effort along the path begun with establishing of the Center for Nuclear Femtography [16].

I am indebted to my collaborators and students: Liliet Calero-Diaz, Michael Engelhardt, Gary Goldstein, Osvaldo Gonzalez-Hernandez, Dustin Keller, Brandon Kriesten, Andy Meyer, Abha Rajan.

\section{References}

[1] A. Accardi et al., Eur. Phys. J. A 52, no. 9, 268 (2016) doi:10.1140/epja/i2016-16268-9 [arXiv:1212.1701 [nucl-ex]].

[2] A. Aprahamian et al., "Reaching for the horizon: The 2015 long range plan for nuclear science," 
[3] X. D. Ji, Phys. Rev. Lett. 78, 610 (1997) doi:10.1103/PhysRevLett.78.610

[4] A. V. Radyushkin, Phys. Rev. D 56, 5524 (1997) doi:10.1103/PhysRevD.56.5524

[5] D. Muller, D. Robaschik, B. Geyer, F. M. Dittes and J. Horejsi, Fortsch. Phys. 42, 101 (1994)

[6] M. Diehl, Phys. Rept. 388, 41 (2003) doi:10.1016/j.physrep.2003.08.002, 10.3204/DESY-THESIS-2003-018

[7] A. V. Belitsky and A. V. Radyushkin, Phys. Rept. 418, 1 (2005) doi:10.1016/j.physrep.2005.06.002

[8] K. Kumericki, S. Liuti and H. Moutarde, Eur. Phys. J. A 52, no. 6, 157 (2016)

[9] A. V. Belitsky, X. d. Ji and F. Yuan, Phys. Rev. D 69, 074014 (2004) doi:10.1103/PhysRevD.69.074014

[10] X. D. Ji, Phys. Rev. D 55, 7114 (1997) doi:10.1103/PhysRevD.55.7114

[11] M. V. Polyakov, Phys. Lett. B 555, 57 (2003) doi:10.1016/S0370-2693(03)00036-4

[12] M. V. Polyakov and A. G. Shuvaev, hep-ph/0207153.

[13] M. V. Polyakov and P. Schweitzer, Int. J. Mod. Phys. A 33, no. 26, 1830025 (2018) doi:10.1142/S0217751X18300259

[14] M. V. Polyakov and P. Schweitzer, arXiv:1812.06143 [hep-ph].

[15] C. Lorcé, H. Moutarde and A. P. Trawinski, Eur. Phys. J. C 79, no. 1, 89 (2019) doi:10.1140/epjc/s10052-019-6572-3

[16] https://pages.shanti.virginia.edu/Femtography/

[17] M. Mazouz et al. [Jefferson Lab Hall A Collaboration], Phys. Rev. Lett. 99, 242501 (2007) doi:10.1103/PhysRevLett.99.242501

[18] J. F. Donoghue, B. R. Holstein, B. Garbrecht and T. Konstandin, Phys. Lett. B 529, 132 (2002) Erratum: [Phys. Lett. B 612, 311 (2005)]

[19] V. D. Burkert, L. Elouadrhiri and F. X. Girod, Nature 557, no. 7705, 396 (2018).

[20] M. Deka et al., Phys. Rev. D 91, no. 1, 014505 (2015)

[21] C. Alexandrou, M. Constantinou, K. Hadjiyiannakou, K. Jansen, C. Kallidonis, G. Koutsou, A. Vaquero AvilÃl's-Casco and C. Wiese, Phys. Rev. Lett. 119, no. 14, 142002 (2017)

[22] P. Hagler et al. [LHPC Collaboration], Phys. Rev. D 77, 094502 (2008)

[23] P. E. Shanahan and W. Detmold, Phys. Rev. D 99, no. 1, 014511 (2019) doi:10.1103/PhysRevD.99.014511

[24] B. P. Abbott et al. [LIGO Scientific and Virgo Collaborations], Phys. Rev. Lett. 119, no. 16, 161101 (2017) doi:10.1103/PhysRevLett.119.161101

[25] S. Liuti, A. Rajan and K. Yagi, arXiv:1812.01479 [hep-ph].

[26] Y. Hatta and D. L. Yang, Phys. Rev. D 98, no. 7, 074003 (2018)

[27] Y. Hatta, A. Rajan and K. Tanaka, JHEP 1812, 008 (2018) doi:10.1007/JHEP12(2018)008

[28] K. Hafidi, S. Joosten, Z. E. Meziani and J. W. Qiu, Few Body Syst. 58, no. 4, 141 (2017). doi:10.1007/s00601-017-1305-3

[29] S. Liuti and S. K. Taneja, Phys. Rev. C 72, 034902 (2005) doi:10.1103/PhysRevC.72.034902 
[30] W. Armstrong et al., arXiv:1708.00888 [nucl-ex].

[31] S. K. Taneja, K. Kathuria, S. Liuti and G. R. Goldstein, Phys. Rev. D 86, 036008 (2012) doi:10.1103/PhysRevD.86.036008

[32] E. R. Berger, F. Cano, M. Diehl and B. Pire, Phys. Rev. Lett. 87, 142302 (2001) doi:10.1103/PhysRevLett.87.142302

[33] S. Liuti, Talk at INT Workshop "Orbital Angular Momentum in QCD", February 6-17, 2012, http://www.int.washington.edu/talks/WorkShops/int_12_49W/

[34] P. Hagler, Phys. Lett. B 594, 164 (2004) doi:10.1016/j.physletb.2004.05.014

[35] R. L. Jaffe and A. Manohar, Nucl. Phys. B 337, 509 (1990). doi:10.1016/0550-3213(90)90506-9

[36] C. Lorce and B. Pasquini, Phys. Rev. D 84, 014015 (2011) doi:10.1103/PhysRevD.84.014015

[37] S. Meissner, A. Metz and M. Schlegel, JHEP 0908, 056 (2009) doi:10.1088/1126-6708/2009/08/056

[38] Y. Hatta, Phys. Lett. B 708, 186 (2012) doi:10.1016/j.physletb.2012.01.024

[39] M. Burkardt, Phys. Rev. D 88, no. 1, 014014 (2013) doi:10.1103/PhysRevD.88.014014

[40] M. Engelhardt, Phys. Rev. D 95, no. 9, 094505 (2017) doi:10.1103/PhysRevD.95.094505

[41] A. Rajan, M. Engelhardt and S. Liuti, Phys. Rev. D 98, no. 7, 074022 (2018) doi:10.1103/PhysRevD.98.074022

[42] A. Rajan, A. Courtoy, M. Engelhardt and S. Liuti, Phys. Rev. D 94, no. 3, 034041 (2016) doi:10.1103/PhysRevD.94.034041

[43] D. W. Sivers, Phys. Rev. D 41, 83 (1990).

[44] M. Engelhardt, P. HÃd'gler, B. Musch, J. Negele and A. SchÃd' fer, Phys. Rev. D 93, no. 5, 054501 (2016)

[45] B. Yoon et al., Phys. Rev. D 96, no. 9, 094508 (2017)

[46] J. w. Qiu and G. F. Sterman, Phys. Rev. Lett. 67, 2264 (1991)

[47] B. Kriesten, S. Liuti, L. Calero-Diaz, D. Keller, A. Meyer, G. R. Goldstein and J. O. Gonzalez-Hernandez, arXiv:1903.05742 [hep-ph].

[48] M. N. Rosenbluth, Phys. Rev. 79, 615 (1950).

[49] M. Defurne et al. [Jefferson Lab Hall A Collaboration], Phys. Rev. C 92, no. 5, 055202 (2015) doi:10.1103/PhysRevC.92.055202 